
abril/junho 2003.

\title{
Selección de progenitores en espárrago
}

\author{
I. Gatti; F. López Anido;L. Picardi; E. Cointry
}

Facultad de Ciencias Agrarias, Universidad Nacional de Rosario. CC 14 - (S2125ZAA) Zavalla. Santa Fe. Argentina. E.mail alegro@arnet.com.ar

\section{RESUMO}

O objetivo principal deste trabalho foi determinar as variáveis que melhor explicam a variação entre indivíduos dentro da população de aspargos originária da cultivar Argenteüil e selecionar plantas superiores para compor novas populações. O ensaio, composto por 1280 plantas, foi instalado no campo experimental da Facultad de Ciencias Agrarias de la Universidad Nacional de Rosario, Santa Fe, Argentina, em 1996. Durante os anos de 1997 e 1998, foram avaliadas as variáveis: número de turiões por planta (TU); peso médio do turião (PM); diâmetro do turião (DI); produção total por planta (RE); produção comercial por planta $(\mathrm{RM})$; número de dias para início da colheita (DAC); número de hastes por planta (NUTA); altura da haste principal (ALT) e peso fresco da massa verde (MASAV). De acordo com a análise de componentes principais, as variáveis RE, RM, PM, DI e TU, são responsáveis por $60 \%$ da variação existente dentro da população avaliada. A análise de agrupamento baseada nestas variáveis, permitiu a identificação de cinco grupos distintos para os dois sexos. Os grupos G5 de plantas estaminadas e G5 de plantas pistiladas $(7,51 \%$ da população total) apresentaram as melhores características de RE e qualidade. Destes grupos, foram selecionadas 6 plantas estaminadas e 8 plantas pistiladas para serem utilizadas como genitores no programa de melhoramento de aspargos.

Palavras-chave: Asparagus officinalis L., componentes principais, análise de agrupamento.

\begin{abstract}
Parent selection in asparagus

The principal aim of this experiment was to ascertain what the variables are which best explain the variations among individuals within the populations of asparagus which originates from the cultivar Argenteüil and as a result select better plants to form new populations. The test, using 1,280 plants, was carried out at he experimental farm in the Facultad Ciencias Agrarias de la Universidad Nacional de Rosario, Santa Fé, Argentina, in 1996. During 1997 and 1998 the number of spears per plant (TU), the mean weight of spears (PM), mean diameter of spears (DI) total yield (RE), marketable yield (RM), days to first harvest (DAC), number of stalks (NUTA), plant height (ALT) and fresh weight of the fern (MASAV) were measured. According to an analysis of the principal components, the variables RE, RM, PM, DI and TU were responsible for $60 \%$ of the variation that exists within the population evaluated.
\end{abstract}

Keywords: Asparagus officinalis L., principal components, cluster analysis

\section{(Recebido para publicação em 22 de agosto de 2001 e aceito em 06 de junho de 2003)}

$E^{1}$ espárrago es una planta dioica perene del género Asparagus, perteneciente a la familia de las Liliáceas. Este género comprende unas 100 especies originarias del sur de Europa, Asia y África, algunas de ellas con valor ornamental y sólo una con valor hortícola (Asparagus officinalis L.).

A lo largo del mejoramiento de esta especie se han desarrollado diferentes tipos de materiales para aumentar el rendimiento y la uniformidad del cultivo. Estos incluyen poblaciones mejoradas por selección masal, distintos tipos de híbridos simples y dobles, híbridos clonales y materiales constituidos por las $\mathrm{F}_{2}$ de híbridos clonales. Independientemente del material a obtener, los programas de mejoramiento siempre incluyen una primera etapa que se refiere a la selección de las plantas elite con las características deseadas. Bannerot et al. (1969) y Bussell et al. (1987) establecieron que el rendimiento de mercado de las dos primeras cosechas está altamente correlacionado con el rendimiento de mercado a más largo plazo, por lo que la etapa de evaluación de planta individual puede concluirse al finalizar el segundo año de cosecha y considerar este período como un buen indicador del futuro rendimiento.

Por otro lado, diversos autores han estudiado las correlaciones fenotípicas existentes entre diversas variables y el rendimiento con el objetivo de identificar aquellas variables de fácil medición que pueden ser utilizadas como criterios de selección. Ellison y Scheer (1959), Ellison et al. (1960) y Chen (1987) destacaron que el rendimiento de plantas individuales de espárrago está correlacionado positivamente con el número de tallos, precocidad y vigor del helecho (masa vegetativa) por lo que estos caracteres pueden ser utilizados como criterios de selección. Así mismo, Silveira \& Agustín (1993) evaluando cultivares e híbridos dioicos llegaron a las mismas conclusiones; pero al evaluar los híbridos "todos macho" (TM) el rendimiento sólo se correlacionó positivamente con el vigor de la planta. Cointry et al. (2000), analizando poblaciones del tipo Argenteüil, establecieron que el número de turiones y el peso promedio de los turiones eran las componentes más importantes del rendimiento y que las variables vegetativas como peso del helecho, número de tallos y altura de planta no presentaron coeficientes de correlación significativos con rendimiento y por lo tanto, al igual que Falloon \& Nikoloff (1986) concluyeron 
que no eran criterios útiles para la selección de plantas elite.

Ellison et al. (1960), Currence \& Richardson (1937) y Ellison (1986) encontraron que el diámetro y el número de turiones están altamente correlacionados con el rendimiento. Sin embargo, según Ellison \& Scheer (1959) y Pandita \& Bhan (1990) el número de turiones se correlaciona negativamente con el diámetro. Estos antecedentes sugieren que para seleccionar mediante estas componentes del rendimiento es necesario realizar una valoración conjunta de ellos para no fijar características negativas en la población asociadas con otros aspectos que han resultado elegidos como criterios de selección.

En este sentido, Punia et al. (1982), Cruz (1990) y Cruz \& Regazzi (1995) afirman que las técnicas estadísticas como el Análisis de Agrupamientos constituyen una herramienta adecuada para el mejor aprovechamiento de las interrelaciones existentes en un grupo de variables, posibilitando la identificación de aquellos genotipos que se destacan del resto. Al realizar un Análisis de Agrupamientos sobre las características individuales de un conjunto de plantas se facilita la identificación de aquellos individuos con la combinación de variables más favorables. Este tipo de análisis fue utilizado por Gatti et al. (2000) para caracterizar siete poblaciones de espárrago con el fin de seleccionar aquellas más apropiadas como fuente de progenitores de híbridos y se encontró que los materiales que presentan interés como fuente de progenitores femeninos no serían los mismos que los de progenitores masculinos. Esta metodología de análisis también fue utilizada por Cointry et al. (2000) con plantas individuales de tres poblaciones del tipo Argenteüil. Las plantas que resultaron selectas mediante este método fueron coincidentes con las plantas elegidas a través de los métodos tradicionales.

El objetivo de este trabajo es aplicar las metodologías de Componentes Principales y Análisis de Agrupamientos para determinar qué variables explican

Tabla 1. Distribución de las variables Rendimiento (RE), Rendimiento de mercado (RM), Peso promedio del turión (PM), Diámetro medio del turión (DI), Número de turiones (TU), Días a cosecha (DAC), Altura de planta (ALT), Número de tallos (NUTA) y Peso de la masa verde (MASAV) que intervienen en cada Componente principal y porcentajes de variancia que explican. Rosário, Facultad de Ciencias Agrarias, 1997/98.

\begin{tabular}{lccc}
\hline \multirow{2}{*}{ Variables } & \multicolumn{3}{c}{ Componente } \\
\cline { 2 - 4 } RE & $\mathbf{1}$ & $\mathbf{2}$ & $\mathbf{3}$ \\
RM & 0,32 & 0,82 & 0,17 \\
PM & 0,58 & 0,65 & 0,25 \\
DI & 0,91 & 0,11 & 0,12 \\
TU & 0,88 & 0,04 & 0,09 \\
DAC & $-0,00$ & 0,89 & 0,23 \\
ALT & 0,36 & 0,18 & $-0,01$ \\
NUTA & 0,39 & 0,00 & 0,70 \\
MASAV & $-0,18$ & 0,34 & 0,75 \\
\hline$\%$ Var. total & 0,11 & 0,20 & 0,83 \\
\hline \% acumulado & 41,60 & 18,04 & 11,51 \\
\hline
\end{tabular}

la variación entre individuos e identificar el grupo de plantas que reúna las mejores características para actuar como progenitoras de un nuevo pool génico para obtener una población mejorada de espárrago blanco tanto para caracteres vegetativos como productivos.

\section{MATERIAL Y MÉTODOS}

Se utilizó una población de 1280 plantas formada por polinización libre entre 32 plantas pistiladas y 64 estaminadas del cultivar Argenteüil, implantada en 1996 en el Campo Experimental J.F. Villarino de la Facultad de Ciencias Agrarias de la Universidad Nacional de Rosario, ubicado en la localidad de Zavalla, provincia de Santa Fe $\left(33^{\circ} 01^{\prime}\right.$ LS y $60^{\circ}$ 53' LO). El ensayo estuvo constituído por 32 familias de medios hermanos, implantadas en un diseño en bloques completos aleatorizados con cuatro repeticiones de 10 plantas por familia. La distancia de plantación fue 2,1 metros entre surcos y 0,45 metros entre arañas dentro del surco. El material fue manejado como espárrago blanco, realizándose el alomado de los surcos antes de la cosecha.

Durante los años 1997 y 1998 se evaluaron las siguientes variables sobre plantas individuales, cosechadas durante un período de 40 días a partir de la aparición del primer turión: Número de Turiones (TU) (cantidad de brotes producidos por cada planta); Peso Medio del turión (PM) (peso promedio del turión en g); Diámetro medio del turión (DI) (diámetro promedio en mm de los turiones de cada planta, medido con calibre en la base del tercio superior del turión); Rendimiento (RE) (peso total en $\mathrm{g}$ de todos los turiones producidos por cada planta); Rendimiento de mercado (RM) (peso total en g de los turiones de cada planta, con diámetro superior o igual a $12 \mathrm{~mm}$ ); Días a cosecha (DAC) (número de días transcurridos desde el 21 de abril (fecha media de corte de la masa aérea) a la primera cosecha de cada planta).

Los turiones se cortaron a $15 \mathrm{~cm}$ de longitud antes de constatar su peso. Luego de la cosecha las plantas se dejaron crecer libremente, y al final del período vegetativo se evaluó: Número de tallos (NUTA) (número total de tallos vegetativos de cada planta); Altura (ALT) (altura en cm del tallo principal de cada planta, tomada desde el nivel del suelo); Masa verde (MASAV) (peso fresco total en g del helecho, cortando cada tallo a nivel del suelo).

Se realizó un análisis multivariado de Componentes Principales (Johnson 


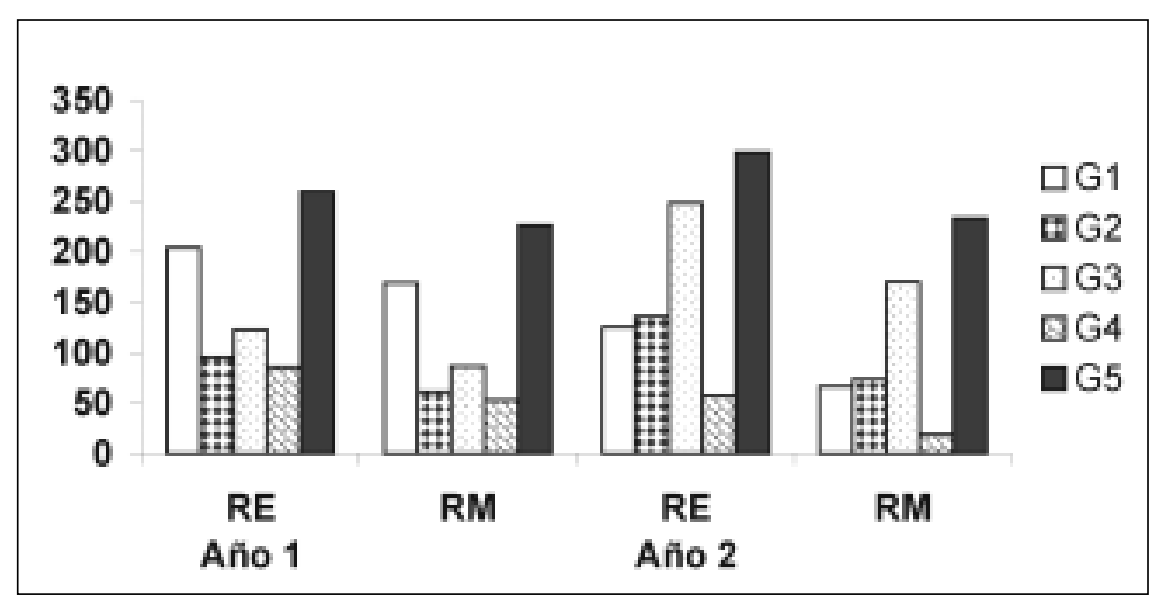

Gráfico 1. Valores medios de Rendimiento (RE) y Rendimiento de Mercado (RM) en cada grupo (G1, G2, G3, G4 y G5) y año en las plantas estaminadas. Rosário, Facultad de Ciencias Agrarias, 1997/98.

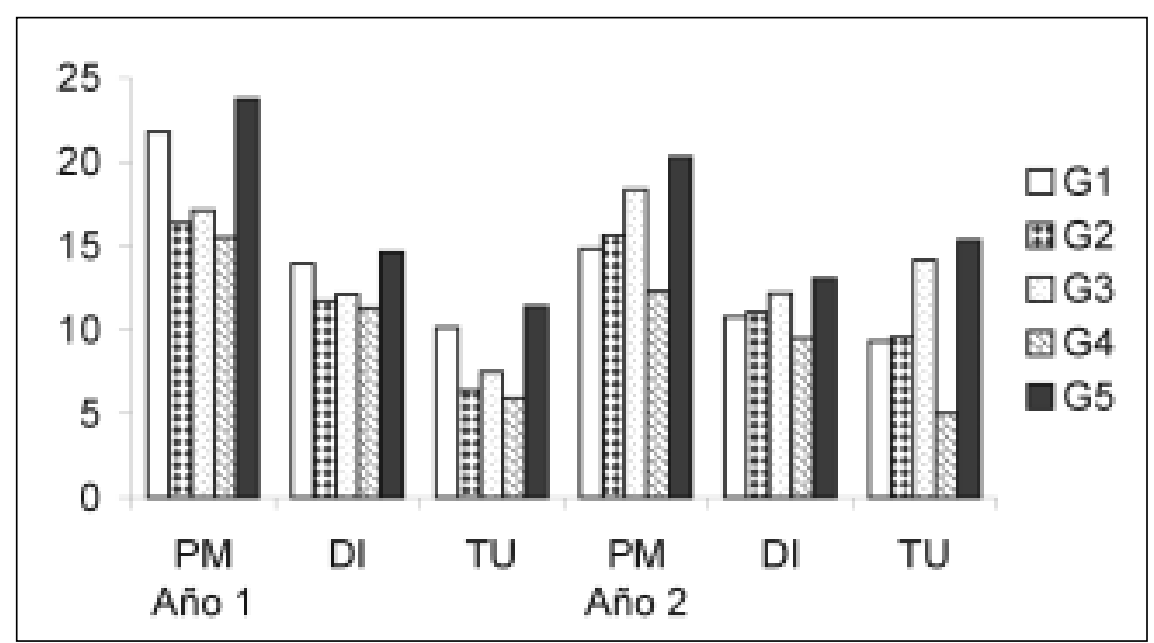

Gráfico 2. Valores medios de Peso promedio del turión (PM), Diámetro medio del turión (DI) y Número de turiones (TU) en cada grupo (G1, G2, G3, G4 y G5) y año en las plantas estaminadas. Rosário, Facultad de Ciencias Agrarias, 1997/98.

\& Wichern, 1998) y un Análisis de Agrupamiento mediante el módulo estadístico Cluster análisis, método Kmeans cluster del programa Statistica (Stat Soft Inc., 1993).

\section{RESULTADOS Y DISCUSIÓN}

El análisis de Componentes principales estableció que los dos primeros componentes explican casi el $60 \%$ de la variación existente (tabla 1). El primer componente, que explicó la variación mayor, estuvo determinado por las variables Peso promedio del turión y Diámetro medio del turión; el segundo componente, por las variables Rendimiento, Rendimiento de mercado y Número de turiones; mientras que el que el porcentaje de plantas que aportaron el $80 \%$ del rendimiento total en tres poblaciones del cultivar Argenteüil fluctuaba alrededor de un $63 \%$ en el primer año de cosecha y un $57 \%$ en el segundo.

En función de las variables determinantes de los dos primeros componentes principales, se realizó un Análisis de Agrupamientos en las plantas estaminadas que permitió conformar 5 grupos con diferencias altamente significativas para todas las variables: G1 integrado por 102 plantas, G2 por 95, G3 por 171, G4 por 186 y G5 por 36 plantas. Los grupos G1 y G4, que comprenden el $48,8 \%$ del total de las plantas estaminadas, muestran en el segundo año de producción una disminución del Rendimiento y el Rendimiento de mercado (grafico 1), explicada por la disminución en los valores de sus componentes, Peso promedio del turión, Diámetro medio y Número de turiones (gráfico 2). Entre ellos, el G4 se caracterizó por presentar en ambos años los menores valores, mientras que el G1 presentaba en el primer año valores relativamente altos que luego se asemejan a los de G2 en el segundo año. El G3 muestra un notable incremento en Rendimiento y Rendimiento de mercado explicado por el incremento en el Número de turiones, ya que tanto Peso promedio como Diámetro medio del turión se mantienen constantes o incrementan levemente.

El G5 es el grupo que presenta mayor interés como material para seleccionar, ya que reúne al $6,1 \%$ de las plantas que presentan los mayores valores de las distintas variables. Este grupo muestra un leve incremento en Rendimiento y Rendimiento de mercado. Puede concluirse que si bien son plantas que disminuyen su Diámetro y Peso promedio del turión en el segundo año, aumentan el Número de turiones, produciendo así similares Rendimientos en ambos años.

Para el caso de las plantas pistiladas, el número de integrantes de cada grupo fue: para el G1, 135; para el G2, 67; para el G3, 141; para el G4, 236 y para el G5, 56.

Los grupos G3, G4 y G5 aumentan su Rendimiento y Rendimiento de mercado en el segundo año de cosecha 
(gráfico 3), debido a un aumento del Número de turiones y, en el caso de G3 y G4, un aumento en el Peso promedio y el Diámetro medio del turión (gráfico 4). el G5 es el grupo que presenta los mayores valores en todas las variables, reuniendo el $8,8 \%$ del total de las plantas pistiladas. Los grupos G1 y G2 muestran una leve disminución en Rendimiento y Rendimiento de mercado debido a una disminución en el Número de turiones.

En la población en conjunto, la fracción formada por el grupo 5 de las plantas estaminadas y el grupo 5 de las pistiladas reune al $7,5 \%$ de 10 s individuos. Estas plantas son las que presentan las mejores características para actuar como progenitoras del siguiente ciclo de selección. Entre ellas fueron elegidas las 8 plantas pistiladas y 6 estaminadas que presentaron mayores Rendimientos.

En función de los valores de heredabilidad en sentido estricto de $0,10 \pm 0,07$ para Rendimiento y 0,13 $\pm 0,07$ para Rendimiento de mercado hallados mediante un análisis de componentes de variancia realizado por Gatti (2001) para la misma población en estudio, se estima que se obtendrá un aumento de $13,4 \%$ en Rendimiento y de $19,4 \%$ en Rendimiento de mercado.

Las variables productivas (Rendimiento, Rendimiento de mercado, Peso promedio del turión, Diámetro medio del turión y Número de turiones) son las que explican el mayor porcentaje de variación entre plantas y permiten diferenciar grupos de individuos con características homogéneas.

Los grupos 5 de plantas estaminadas y 5 de pistiladas $(7,51 \%$ de la población total) presentan las mejores características de Rendimiento y calidad.

\section{LITERATURA CITADA}

BANNEROT H.; DERIEUX, M.; THEVENIN, L.; ARNOUX, J. Résultats de'un essai comparatif de populations d' asperge. Annales Amélioration des Plantes v. 19, n. 3, p. 289-324, 1969.

BUSSELL, W.T.; FALLOON, P.G.; NIKOLOFF, A.S. Evaluation of asparagus yield performance after two years' harvesting. New Zealand Journal of Experimental Agronomies, v. 15, p. 205-208, 1987. CHEN, Y.W.; HUNG, L.; TU, C.C. Use of economic characters as ideotypes in asparagus breeding. Proceeding of the Breeding of Horticultural Crops, v. 35, p. 45-49, 1987.

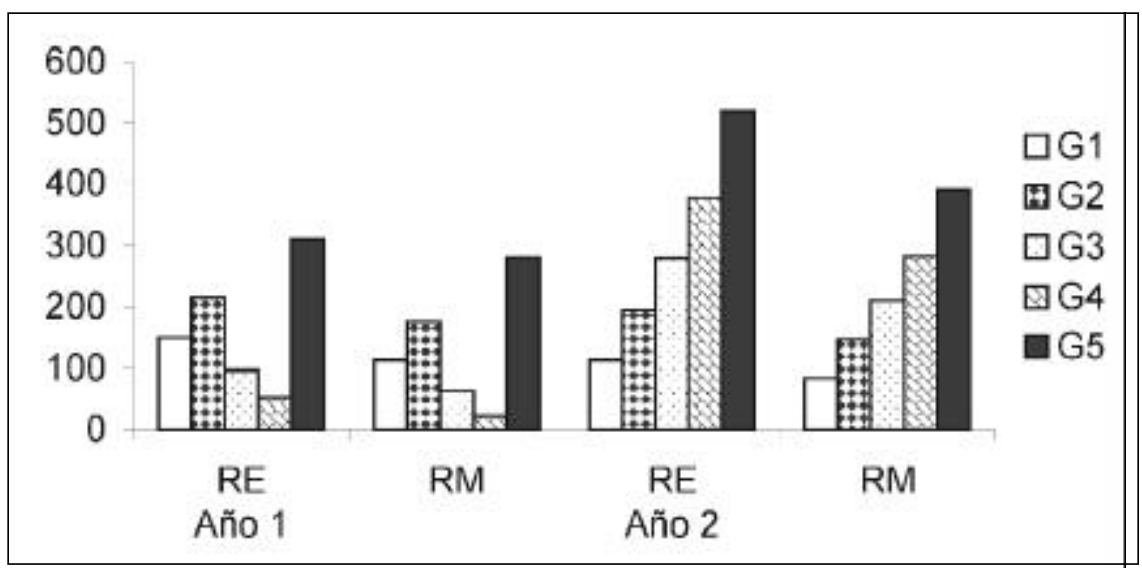

Gráfico 3. Valores medios de Rendimiento (RE) y Rendimiento de Mercado (RM) en cada grupo (G1, G2, G3, G4 y G5) y año en las plantas pistiladas. Rosário, Facultad de Ciencias Agrarias, 1997/98.

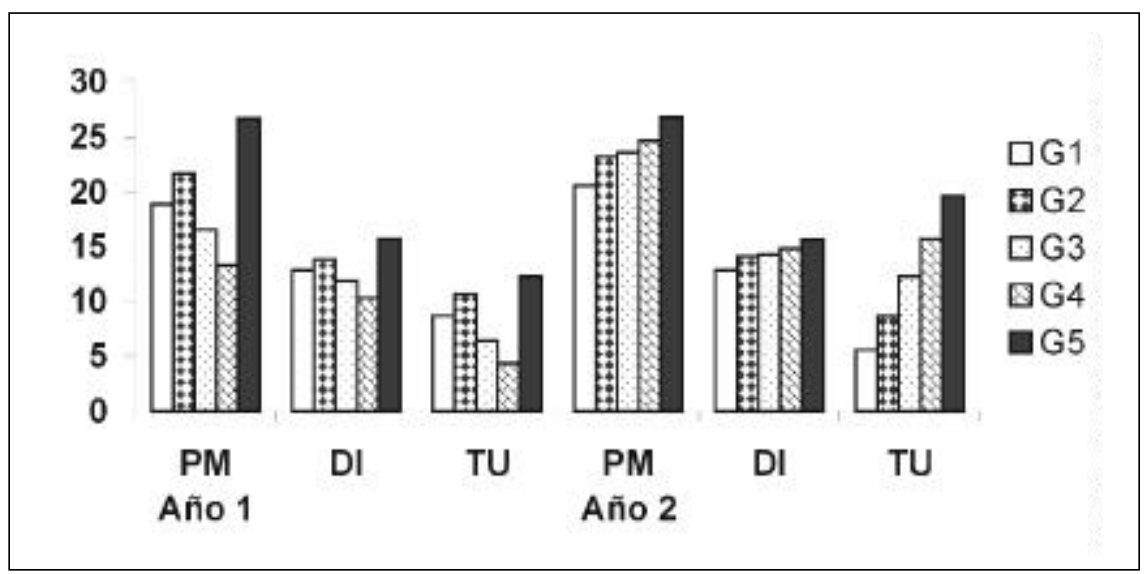

Gráfico 4. Valores medios de Peso promedio del turión (PM), Diámetro medio del turión (DI) y Número de turiones (TU) en cada grupo (G1, G2, G3, G4 y G5) y año en las plantas pistiladas. Rosário, Facultad de Ciencias Agrarias, 1997/98.

COINTRY, E; LÓPEZ ANIDO, F.S; GATTI, I.; CRAVERO, V.P.; FIRPO, I.T.; GARCÍA, S.M. Early selection of elite plants in asparagus. Bragantia, Campinas, v. 59, n. 1, p. 21-26, 2000. CRUZ, C.D. Aplicação de algumas técnicas multivariadas no melhoramento de plantas. Piracicaba: USP/ESLAQ, 1990. 188 p. (Tese doutorado)

CRUZ, C.D.; REGAZZI, A.J. Modelos biométricos aplicados no melhoramento genético. Viçosa: UFVImprensa Universitária, 1995. 390 p.

CURRENCE, T.M.; RICHARDSON, A.L. Asparagus breeding studies. Proceedings of American Society of Horticultural Science, v. 35, p. 554-557, 1937.

ELLISON, J.H. Asparagus breeding. In: BASSETT, M.J., ed. Breeding Vegetable Crops. Westport CT, AVI, Avi Publisher Co., 1986. p. 521-569.

ELLISON, J.H.; SCHEER, D.F.; WAGNER, J.J. Asparagus yield as related to plant vigor, earliness and sex. Proceedings of American Society of Horticultural Science, v. 75, p. 411-415, 1960. ELLISON, J.H.; SCHEER, D.F. Yield related to brush vigor in asparagus. Proceedings of American Society of Horticultural Science, v. 73, p. 339-344, 1959.
GATTI, I.; CRAVERO, V.; LÓPEZ ANIDO, F.; COINTRY, E. Evaluación de siete poblaciones de espárrago (Asparagus officinalis L.). Pesquisa Agropecuária Brasileira, Brasília, v. 35, n. 6, p. $1151-1157,2000$

GATTI, I. Selección de progenitores y cálculo de parámetros genéticos en espárrago (Asparagus officinalis L.). Rosario: Facultad de Ciencias Agrarias, 2001.81 p. (Tese mestrado)

JOHNSON, A.R.; WICHERN, D.W. Applied multivariate statistical analysis. 4. ed. New Jersey: Upper Sadle River, 1999. 816 p.

PANDITA, P.N.; BHAN, M.K. Variability and correlation in asparagus Asparagus officinalis). Indian Journal of Agricultural Sciences, v. 60, n. 7, p. 487-488, 1990

PUNIA, M.S.; HOODA, R.S.; PARODA, R.S. Discriminant function analysis for cane-yield attributes in sugarcane. Indian Journal of Genetics and Plant Breeding, v. 52, p. 643-645, 1982. ROBB, O.J. Some observation on individual asparagus plants records. Scientific Agriculture, v. 17, n. 3, p. 144-145, 1936.

SILVEIRA, R.F.; AUGUSTIN, E. Relações entre diâmetro e número de hastes, altura e vigor de plantas e produtividade de aspargo. Pesquisa Agropecuaria Brasileira, Brasília, v. 28, n. 1, p. 29-133, 1993.

STATISTICS CLUSTER ANALYSIS, StatSoft, Inc. 1993. 